department or organisational issues. An action plan was develop to address the lowest scoring areas.

The survey was completed again in 2015 demonstrating similar positive results achieving Level 4 again with a score of 61.05 out of 69 .

\section{P-269 EMBRACING CHANGE: FOCUSING ON OUR PEOPLE, OUR PARTNERSHIPS AND OUR POTENTIAL, ARTHUR RANK HOSPICE CHARITY: PART 2 OF OUR JOURNEY}

Liz Webb. Arthur Rank Hospice Charity, Cambridge, UK

\subsection{6/bmjspcare-2016-001245.288}

Arthur Rank Hospice Charity is on a dual journey of moving from the NHS to independence and building and moving to a new hospice building. This presented us with a double dose of significant change for our staff in the space of 18 months.

We recognised that we have an opportunity to shape and transform our organisational culture as a new team- a mixture 100 plus NHS staff and 20 previous charity staff. At the same time as embedding the NICE pathway-Workplace health policy and management practices (2015). Managing the changes taking place with our staff is vital to the ongoing success of the charity and quality of care that we provide.

What we did

Pre TUPE of 100 NHS staff

- Charity CEO and NHS Clinical lead worked together to communicate clearly and robustly with both teams about what was happening and when.

- Occupational health support and 24/7 access to counselling.

\section{At point of integration of the two previous teams}

- Effective communication. Programme of face to face meetings with the new senior leadership team:

- Communicated a new quality governance structure that supports information flow from bottom to top of the organisation.

- Commissioned a series of cultural workshops with all staff over 10 weeks. All staff were invited to the full day events and all attended except three.

\section{Outcomes of workshops and next steps}

Using a model of 'appreciative enquiry' the workshops identified where we were and what steps we needed to take to embed a culture of openness, transparency, innovation and change.

A plan of work was developed to support the new culture and focus on wellbeing with the now integrated staff team.

This includes

- Fortnightly bulletin

- Staff Forum

- clinical supervision for all clinical staff.

- 1.1 meetings with staff and line manager

- annual appraisal and clear objectives

- Research based journal club

- Closing the loop', ensuring information and outcomes from projects, investigations and complaints and shared with all.

- Move to new building-program of includes a team approach to resilience and change management.
P-270 THE MACMILLAN VALUE BASED STANDARDS: HOW A HOSPICE TOOK MACMILLAN VALUES TO ITS HEART

Sandie Robinson, Roger Wheelwright. John Taylor Hospice, Birmingham, UK

10.1136/bmjspcare-2016-001245.289

How a hospice adopted a culture of care by using Macmillan Values Based Standard (MVBS) where a patient and their loved ones are respected and valued. Fostering positive attitudes with staff to help shape services and reflect on the quality of their care.

MVBS were developed to bridge the gap between the principles associated with person-centred care and the behaviours and expectations that should form part of healthcare practices.

The eight standards set out behaviours with front line staff which are at the centre of driving forward standards of service. Staff awareness of the MVBS, allowing ideas and innovations to improve care from the bottom up. Staff engagement is essential to allow change and improvement to care provision and sustainability of a quality service. Clinical supervision of staff, enables teams to analyse situations and suggest different ways of working. The reflection process enables conceptualisation of how they can integrate the fundamental values and behaviours set out in the MVBS within their everyday practice.

Team meetings recap on standards focussing on one standard each month to remind staff how the standard can be implemented into their working day. Feedback from service user experience is at the heart of what we do and essential to evaluate care and prevent disconnection. One main measure from any individual within a hospice organisation is to question 'have I made a difference to that individual?" "How we care for the dying is an indicator of how we care for all sick and vulnerable people".

Using the MVBS to set our standards of care as an organisation allows our staff to measure themselves individually and as a team. Through processes of reflection, clinical supervision, peer support and real time feedback we can continue to strive for excellence in care and influence change and improvement to our service.

\section{P-271 OPTIMISING TEAM WORKING IN WIGAN \& LEIGH HOSPICE NURSE SPECIALIST SERVICE}

Jenny Gallagher, Rebecca Lennon. Wigan and Leigh Hospice, Wigan, UK

\subsection{6/bmjspcare-2016-001245.290}

Introduction Effective teams improve quality, whilst reducing staff stress levels. The degree of self-rated teamwork is also related to patient and carer satisfaction. Teams do not work just because they exist and conflict is both inevitable and healthy. We undertook a review of our own team working strategies with the aim to improve collaborative working, inter and intra-team communication, time management and understand the diversity within our team. Overall, we aimed to uncover potential and identify opportunities to develop.

What did we do?

- Completed an anonymised survey evaluating how the team currently functions.

- Developed a purpose statement and operating principles.

- Team service objectives were reviewed.

- Time management training. 